\title{
A Research on the Combined Maintenance Strategy for Production Line Equipment Based on Mixed Failure Rate
}

\author{
Binbin Xu $\mathbb{D}^{1,2}$ Ziyue Wang $\mathbb{D}^{1},{ }^{1}$ Wei Luo $\mathbb{D}^{1},{ }^{1}$ Tianqi Ma $\mathbb{D}^{1},{ }^{1}$ and Hao Huang $\mathbb{D}^{1}$ \\ ${ }^{1}$ Key Laboratory of CNC Equipment Reliability, Ministry of Education, School of Mechanical and Aerospace Engineering, \\ Jilin University, Changchun 130022, China \\ ${ }^{2}$ Sino-German College of Intelligent Manufacturing, Shenzhen Technology University, Shenzhen 518118, China
}

Correspondence should be addressed to Wei Luo; luoweicn@jlu.edu.cn

Received 24 September 2020; Revised 26 January 2021; Accepted 5 February 2021; Published 18 February 2021

Academic Editor: Akhil Garg

Copyright (C) 2021 Binbin Xu et al. This is an open access article distributed under the Creative Commons Attribution License, which permits unrestricted use, distribution, and reproduction in any medium, provided the original work is properly cited.

In order to improve the reliability of the production line and reduce its maintenance costs, this paper establishes preventive maintenance (PM) models of the equipment, which are classified into different levels based on mixed failure rate. The differential and targeted maintaining strategies are formulated as well. First, a method for dividing important equipment and secondary equipment of a production line based on grey interval Analytic Hierarchy Process- (AHP-) Entropy method is proposed. Next, the dynamic service age regression factor and the dynamic failure rate increasing factor are combined to establish a mixed failure rate model. Finally, for the important equipment and secondary equipment, two PM models are established with the constraint of reliability, the objective of maximum availability, and minimum maintenance cost rate to determine the optimal number of PM and cycles. Combined with an example of production line equipment, the optimal maintenance strategies for important equipment and secondary equipment are obtained. The result verified the feasibility of the model, which effectively improved the reliability of the production line equipment and reduced maintenance costs.

\section{Introduction}

Equipment is an important part of the production line, and maintenance is important for the equipment normal operation. Choosing a reasonable maintenance method will directly affect the production efficiency and product quality of the production line and influence the enterprise economic benefits. Therefore, it is very important for the enterprise to propose a scientific and effective method to divide the production line equipment. Enterprises can implement key management, formulate effective maintenance strategies, rationally allocate related resources, and improve the equipment reliability and maintainability according to the division level.

Multi-index comprehensive evaluation is a common method to solve equipment division. According to different weight determination methods, it can be divided into three categories: The first is subjective weighting method, such as AHP [1, 2], Delphi method [3], and fuzzy comprehensive evaluation method [4]. This method is greatly influenced by human factors with subjective judgment color. The second is objective weighting method, such as principal component analysis [5], Entropy method [6], and TOPSIS method [7]. This method depends on sufficient sample data to ignore the relative importance of experts. The third is the combination weighting method $[8,9]$, which can make the experts to weight different attributes achieve the subjective and objective unity. Experts will use interval numbers to describe them for incomplete information and complex problems. All the above studies use a single method or a simple combination of the two methods. It is of great significance to propose a weight determination method that considers the interval numbers and combination weighting method. This method can not only consider the advantages and disadvantages of subjective and objective weighting methods but also accurately reflect the actual situation of decision information.

Maintenance is an important means to ensure the equipment normal operation. Scientific maintenance strategies can improve equipment reliability and reduce maintenance costs. The maintenance methods are divided into 
breakdown maintenance (BM), PM, and RM. PM refers to the periodic systematic inspection of equipment to prevent failures. In this paper, the PM is a state of imperfect PM. It is assumed that the equipment state after PM is between complete maintenance and minimum maintenance; that is, "repair is not new." This method can reduce or eliminate the occurrence of equipment failure, but it will increase unnecessary downtime and easily cause overrepair or underrepair. In the related research on maintenance strategy optimization, the concept of imperfect PM is the most widely used. Zhou et al. [10] proposed a reliability-centric PM strategy for continuous monitoring of degraded systems due to imperfect maintenance and performed imperfect PM. Sheu et al. [11] put forward a generalized periodic imperfect PM model for a system with age-related failure types. Lin et al. [12] proved that, for degenerated complex repairable systems, PM strategies based on aperiodic conditions with different condition variables are usually more effective than those based on periodic life. Yang et al. [13] studied a novel two-phase PM strategy for a single-component system. The two phases are the imperfect maintenance phase and the delayed replacement phase. In recent years, Xia et al. [14-16] have done indepth research in the field of production line maintenance strategy. Based on the PM model, the sensor-driven forecasting method and opportunistic maintenance strategy are combined, and a new opportunistic maintenance strategy is developed to deal with the complex series-parallel structure of the production line. All researches can effectively reduce maintenance costs, avoid system failures, and ensure product quality. BM refers to the repair after the equipment failure and the equipment status is "repair as old," which is simple and of low cost. The equipment status after RM is "repair as new," which can completely eliminate hidden troubles, but of high cost. BM and RM can be regarded as the two extreme states of PM. Therefore, in order to ensure that the proposed maintenance strategy is more accurate, this paper adopts three maintenance methods: BM, PM, and RM.

$\mathrm{PM}$ can be divided into periodic PM and variable cycle $\mathrm{PM}$ according to different maintenance cycle. It is more convenient to implement the periodic PM, but it is difficult to achieve the best preventive effect in actual production. After each PM, the equipment will not return to a new state, and the failure rate will not drop to zero. Therefore, it is difficult to achieve the best preventive effect. It is more reasonable to adopt the variable cycle PM. The age improvement and failure rate changes of equipment after PM are often described by introducing virtual age reduction factor and incremental failure rate factor. Malik [17] and Nakagawa [18] have proposed the concepts of virtual age and failure rate to describe the changes before and after equipment maintenance, laying a theoretical foundation for the equipment PM model. Then, many scholars have conducted in-depth research in this field [19-22]. Lim and Park [23] proposed a periodic PM model considering the number of maintenances and virtual age reduction factor. The virtual age reduction factor only considered the impact of maintenance costs and life. Qu et al. [24] considered the equipment age and the maintenance costs and set the general sequential PM model and the phasic sequential PM model by minimizing the cost rate of the maintenance in the life cycle of the system. Zhuo et al. [25] considered the virtual age reduction factor related to the PM costs, virtual age, and learning effect in the process of maintenance and established a maintenance strategy model aiming at minimizing the expected costs. These studies only considered 2-4 parameters when establishing the failure rate model, which was not comprehensive for the production line equipment, and further research is needed.

This paper takes production line equipment as the research object. First, the grey interval AHP-Entropy method is used to comprehensively evaluate and divide all the evaluated equipment. Next, a combined maintenance method of BM, $\mathrm{PM}$, and RM is proposed. A mixed failure rate model is established by introducing a dynamic virtual age reduction factor with multiple factors and a dynamic incremental failure rate factor considering the number of PM. Finally, for the important equipment and the secondary equipment, two PM models are established with the reliability as the constraint, the maximum availability, and the minimum maintenance cost rate as the optimization objective to determine the optimal number of PM and cycles. The model is verified with an example of production line equipment.

\section{Equipment Division Based on Grey Interval AHP-Entropy Method}

2.1. Determining Index Combination Weights for Interval AHP-Entropy Method. Interval AHP-Entropy method [26] is a weight determination method that effectively combines the interval number with AHP and Entropy method. It not only makes use of the advantages of combination weighting method to achieve the unity of subjective and objective index evaluation but also effectively solves the problem of evaluation uncertainty. It is a more scientific and comprehensive index evaluation method.

In this paper, the interval number is combined with AHP and Entropy method, respectively, to obtain interval AHP [27] and interval Entropy method [28]. Assume that the subjective and objective weight interval ranges of indexes are $w_{A H P}=\left[w_{a}, w_{b}\right]$ and $w_{E M}=\left[w_{x_{i}}, w_{y_{i}}\right]$, and the weight interval range after combination is denoted as $w_{i}=\left[w_{i}^{-}, w_{i}^{+}\right]$.

$$
\begin{aligned}
& w_{i}^{-}=\frac{w_{a} \cdot w_{x_{i}}}{\sum_{i=1}^{m} w_{a} \cdot w_{x_{i}}}, \\
& w_{i}^{+}=\frac{w_{b} \cdot w_{y_{i}}}{\sum_{i=1}^{m} w_{b} \cdot w_{y_{i}}} .
\end{aligned}
$$

Take the intermediate value of each interval as the combined weight $w_{i}$.

$$
W_{i}=\frac{w_{i}^{-}+w_{i}^{+}}{2}
$$

\subsection{Determining Index Weights for Interval AHP}

2.2.1. Establishing a Hierarchy Model. An equipment importance evaluation index degree is established. The first 
layer is the production line equipment importance evaluation index $U$. The second layer $U_{i}$ includes five evaluation indexes of operating rate, reliability, maintainability, economy, and detectability, which are expressed by $\left\{U_{1}, U_{2}, U_{3}, U_{4}, U_{5}\right\}$

2.2.2. Constructing Interval AHP Judgment Matrix. Each element in the judgment matrix is an interval number; assume that $U_{i i}=[1,1], \quad U_{i j}=\left[a_{i j}, b_{i j}\right] U_{i j}=1 / u_{i j}=$ $\left[\left(1 / b_{i j}\right),\left(1 / a_{i j}\right)\right], i, j=1,2, \ldots, m ; a_{i j}$ and $b_{i j}$ are the upper and lower limits of the judgment result. The value of the judgment element adopts the 1-9 scale and its reciprocal scale method. Ask $k$ experts to rate the evaluation index, and let $\quad a_{i j}=(1 / k) \sum_{k=1}^{k} a_{i j}^{(k)} \quad$ and $\quad b_{i j}=(1 / k) \sum_{k=1}^{k} b_{i j}^{(k)}$, $1 / 9 \leq a_{i j} \leq b_{i j} \leq 9$. The interval number judgment matrix is obtained as

$$
U=\left[\begin{array}{cccc}
{[1,1]} & {\left[a_{12}, b_{12}\right]} & \ldots & {\left[a_{1 m}, b_{1 m}\right]} \\
{\left[\frac{1}{b_{12}}, \frac{1}{a_{12}}\right]} & {[1,1]} & \ldots & {\left[a_{2 m}, b_{2 m}\right]} \\
\ldots & \ldots & \ldots & \ldots \\
{\left[\frac{1}{b_{1 m}}, \frac{1}{a_{1 m}}\right]\left[\frac{1}{b_{2 m}}, \frac{1}{a_{2 m}}\right]} & \ldots & {[1,1]}
\end{array}\right] .
$$

2.2.3. Determining the Range of Index Weights. $A=\left(a_{i j}\right)_{m \times m}, B=\left(b_{i j}\right)_{m \times m}$, and the interval logarithm leastsquare method [29] is used for $A$ and $B$ to obtain the normalized weight vectors $w_{l}$ and $w_{u}$, respectively:

$$
\begin{gathered}
w_{l}=\frac{\left[\prod_{i=1}^{m}\left(a_{k i} a_{k k} / a_{i k} a_{i i}\right)\right]^{(1 / 2 m)}}{\sum_{j=1}^{m} \prod_{i=1}^{m}\left(a_{j i} a_{j j} / a_{i j} a_{i i}\right)^{(1 / 2 m)}}, \quad k=1,2, \ldots, m, \\
w_{u}=\frac{\left[\prod_{i=1}^{m}\left(b_{k i} b_{k k} / b_{i k} b_{i i}\right)\right]^{(1 / 2 m)}}{\sum_{j=1}^{m} \prod_{i=1}^{m}\left(b_{j i} b_{j j} / b_{i j} b_{i i}\right)^{(1 / 2 m)}}, \quad k=1,2, \ldots, m .
\end{gathered}
$$

The index weight range of interval AHP is

$$
w_{A H P}=\left[w_{a}, w_{b}\right]=\left[k_{l} w_{l}, k_{u} w_{u}\right],
$$

where $k_{l}=\sqrt{\sum_{j=1}^{m} 1 / \sum_{i=1}^{m} b_{i j}}, k_{u}=\sqrt{\sum_{j=1}^{m} 1 / \sum_{i=1}^{m} a_{i j}}$, and $k_{l}$ and $k_{u}$ are all positive real numbers satisfying $0<k_{i} w_{i}<k_{u} w_{u}$.

\subsection{Determining Index Weights for Interval Entropy Method}

2.3.1. Indexes Normalization. Normalize matrices A and B according to equations (6) to (9). For positive indexes,

$$
\begin{aligned}
a_{i j}^{\prime} & =\frac{a_{i j}-\min \left\{a_{i 1}, a_{i 2}, \ldots, a_{i m}\right\}}{\max \left\{a_{i 1}, a_{i 2}, \ldots, a_{i m}\right\}-\min \left\{a_{i 1}, a_{i 2}, \ldots, a_{i m}\right\}}, \\
b_{i j}^{\prime} & =\frac{b_{i j}-\min \left\{b_{i 1}, b_{i 2}, \ldots, b_{i m}\right\}}{\max \left\{b_{i 1}, b_{i 2}, \ldots, b_{i m}\right\}-\min \left\{b_{i 1}, b_{i 2}, \ldots, b_{i m}\right\}},
\end{aligned}
$$

and, for negative indexes,

$$
\begin{aligned}
a_{i j}^{\prime} & =\frac{\max \left\{a_{i 1}, a_{i 2}, \ldots, a_{i m}\right\}-a_{i j}}{\max \left\{a_{i 1}, a_{i 2}, \ldots, a_{i m}\right\}-\min \left\{a_{i 1}, a_{i 2}, \ldots, a_{i m}\right\}}, \\
b_{i j}^{\prime} & =\frac{\max \left\{b_{i 1}, b_{i 2}, \ldots, b_{i m}\right\}-b_{i j}}{\max \left\{b_{i 1}, b_{i 2}, \ldots, b_{i m}\right\}-\min \left\{b_{i 1}, b_{i 2}, \ldots, b_{i m}\right\}} .
\end{aligned}
$$

2.3.2. Determining the Information Entropy Weight of Each Evaluation Index. Calculate the proportion of the $j$-th value in the lower and upper limits under the $i$-th index according to the two following equations:

$$
\begin{gathered}
g_{i j}=\frac{a_{i j}^{\prime}}{\sum_{j=1}^{m} a_{i j}^{\prime}}, \\
h_{i j}=\frac{b_{i j}^{\prime}}{\sum_{j=1}^{m} b_{i j}^{\prime}} .
\end{gathered}
$$

The information Entropy values of the lower and upper limits of the $i$-th index are

$$
\begin{aligned}
& x_{i}=-\frac{1}{\ln m} \sum_{j=1}^{m} g_{i j} \ln g_{i j}, \\
& y_{i}=-\frac{1}{\ln m} \sum_{j=1}^{m} h_{i j} \ln h_{i j} .
\end{aligned}
$$

If $g_{i j}=0, h_{i j}=0$, record $x_{i}=0, y_{i}=0$.

The index weight range of the interval Entropy method is

$$
w_{E M}=\left[w_{x_{i}}, w_{y_{i}}\right],
$$

where $w_{x i}=1-x_{i} / m-\sum_{i=1}^{m} x_{i}$ and $w_{y i}=1-y_{i} / m-\sum_{i=1}^{m} y_{i}$.

2.4. Establishing Grey Evaluation Model. The grey evaluation method $[30,31]$ uses the grey theory to process the dispersed information of experts into a grey evaluation matrix and processes the single value to obtain the comprehensive evaluation value of the evaluated equipment, which can be used for sorting and optimizing the evaluated results. The comprehensive evaluation method combining interval AHP-Entropy method and grey evaluation method is applied to the division of production line equipment, which can make full use of all basic data and improve the scientific 
accuracy of evaluation results. The specific calculation steps are as follows.

2.4.1. Determining the Evaluation Sample Matrix. Assume that $k$ experts scored the equipment $s$ according to the evaluation index $U_{i}$, and the score is recorded as $d_{i k}^{(s)}$. Arrange the scores into a matrix form, and record the evaluation sample matrix of the $s$-th evaluated equipment as $D^{(s)}=d_{i k}^{(s)}, i=1,2, \ldots, m$.

2.4.2. Determining the Grey Category and Whitening Weight Function. Assume that the grey category is $e(e=1,2, \ldots, p)$, and the three commonly used whitening weight function expressions are as follows:

(1) The upper whitening weight function $f_{1}$ :

$$
f_{1}\left(d_{i k}^{(s)}\right)=\left\{\begin{array}{l}
\frac{d_{i k}^{(s)}}{d_{1}}, d_{i k}^{(s)} \in\left[0, d_{1}\right], \\
1, d_{i k}^{(s)} \in\left[d_{1}, \infty\right], \\
0, d_{i k}^{(s)} \notin[0, \infty] .
\end{array}\right.
$$

(2) The medium whitening weight function $f_{2}$ :

$$
f_{2}\left(d_{i k}^{(s)}\right)=\left\{\begin{array}{l}
\frac{d_{i k}^{(s)}}{d_{1}}, d_{i k}^{(s)} \in\left[0, d_{1}\right], \\
\frac{d_{2}-d_{i k}^{s)}}{d_{2}-d_{1}}, d_{i k}^{(s)} \in\left[d_{1}, d_{2}\right], \\
0, d_{i k}^{(s)} \notin\left[0, d_{2}\right] .
\end{array}\right.
$$

(3) The lower whitening weight function $f_{3}$ :

$$
f_{3}\left(d_{i k}^{(s)}\right)=\left\{\begin{array}{l}
1, d_{i k}^{(s)} \in\left[0, d_{1}\right], \\
\frac{d_{2}-d_{i k}^{(s)}}{d_{2}-d_{1}}, d_{i k}^{(s)} \in\left[d_{1}, d_{2}\right] \\
0, d_{i k}^{(s)} \notin\left[0, d_{2}\right] .
\end{array}\right.
$$

2.4.3. Determining the Grey Evaluation Coefficient. For the evaluation index $U_{i}$, the grey evaluation coefficient $\sigma_{i e}^{(s)}$ of $U_{i}$ belonging to the $e$-th grey category and the total grey evaluation coefficient $\sigma_{i}^{(s)}$ belonging to each grey category are

$$
\begin{aligned}
& \sigma_{i e}^{(s)}=\sum_{k=1}^{k} f_{e}\left(d_{i k}^{(s)}\right), \\
& \sigma_{i}^{(s)}=\sum_{e=1}^{p} \sum_{k=1}^{k} f_{e}\left(d_{i k}^{(s)}\right) .
\end{aligned}
$$

2.4.4. Determining the Grey Evaluation Weight Vector and Matrix. For the evaluation index $U_{i}$, the grey evaluation weight for the $s$-th evaluation equipment of the $e$-th grey category is $r_{i e}^{(s)}$ and the grey evaluation weight vector for each grey category is $r_{i}^{(s)}$ :

$$
\begin{aligned}
& r_{i e}^{(s)}=\frac{\sigma_{i e}^{(s)}}{\sigma_{i}^{(s)}}, \\
& r_{i}^{(s)}=\left(r_{i 1}^{(s)}, r_{i 2}^{(s)}, \ldots, r_{i p}^{(s)}\right) .
\end{aligned}
$$

Summarize the indexes of all the evaluated devices, and obtain the grey evaluation weight matrix of the evaluation index $U$ for the $s$-th evaluated equipment as $R^{(s)}=\left(r_{1}^{(s)}, r_{2}^{(s)}, \ldots r_{k}^{(s)}\right)$

2.4.5. Comprehensive Evaluation of $U$. The comprehensive evaluation result of $U$ for the $s$-th evaluated equipment is $C^{(s)}$.

$$
C^{(s)}=W \cdot R^{(s)}
$$

where $W$ is a combination weight vector of each evaluation index $U_{i}$.

2.4.6. Comprehensive Evaluation Value and Ranking for Production Line Equipment. Assume that the grey evaluation values are $d_{1}, d_{2}, \ldots d_{p}$, vector is $E=\left(d_{1}, d_{2}, \ldots d_{p}\right)$, and the comprehensive evaluation value of the $s$-th evaluated equipment is $Z^{(s)}$.

$$
Z^{(s)}=C^{(s)} \cdot E^{T}
$$

Finally, the evaluated equipment is sorted according to the size of the comprehensive evaluation value and classified by the ABC classification method. Assume that the top $20 \%$ of equipment is defined as important equipment, $20 \%-50 \%$ of equipment is defined as secondary equipment, and the remaining equipment is defined as general equipment.

\section{Formulating Maintenance Strategies for Production Line Equipment}

This paper divides the production line equipment into important equipment, secondary equipment, and general equipment. Considering the above three maintenance 
methods, this paper proposes a combined maintenance strategy for production line equipment. The combined maintenance strategy is multiple BM, $n$ - $1 \mathrm{PM}$, and $n$-th RM in one equipment maintenance replacement cycle. Within $n$ 1 maintenance cycles, the equipment performance gradually degrades, and PM is adopted. In the $n$-th maintenance cycle, $\mathrm{RM}$ is adopted due to poor improvement effect. In the interval of PM, BM should be carried out in case of failure. The specific combined maintenance strategy is described in Figure 1.

In Figure 1, $T$ is a preventive maintenance replacement cycle of the equipment, $\tau_{i}(i=1,2, \ldots, n)$ is the PM cycle, $k_{i}$ is the number of BM in each PM cycle, $\tau_{d}$ is the average maintenance time of $\mathrm{BM}, \tau_{\mathrm{pm}}$ is the average maintenance time of PM, and $\tau_{r}$ is the average duration time of RM.

For the production line, the important equipment has a great impact on it. Once the equipment stops, it will seriously affect the production cycle and may even threaten the environment and personal safety. The impact of secondary equipment on it is lower than the important equipment. Enterprises are more concerned about lowest costs. However, only pursuing the lowest costs may cause frequent equipment failures, which will also have a greater impact. Therefore, the production line equipment maintenance strategy is to adopt a combined maintenance method for both important and secondary equipment and establish preventive maintenance models with reliability as the constraint and the availability and maintenance cost rate as the objective functions to determine the optimal number of PM $n$ and the PM cycle $\tau_{i}$ in the combined maintenance strategy. For general equipment, BM is adopted.

\section{Establishing the PM Model for Production Line Equipment}

4.1. Mixed Failure Rate Model for Equipment. The equipment failure rate distribution function is related to the PM model. In order to make the failure rate model more realistic, this paper introduces a dynamic virtual age reduction factor and a dynamic incremental failure rate factor to describe the equipment change of age and failure rate after PM.
The virtual age reduction factor refers to the degree of age regression. In this paper, $\gamma_{i}$ is used to represent the virtual age reduction factor for the $i$-th PM. $\gamma_{i}$ is a dynamic value. A larger virtual age reduction factor indicates that the equipment remaining life becomes larger after PM. The PM cost, RM cost, equipment age, number of PM, maintenance learning effect, and personnel experience factors are introduced to describe the age change after maintenance, making the virtual age reduction factor model more accurate and consistent with the actual situation. Its expression is

$$
\gamma_{i}=\left(\eta \frac{C_{\mathrm{pm}}}{C_{r}}\right)^{q i \cdot(i+c)^{v} \cdot n^{z}}, \quad 0 \leq \gamma_{i} \leq 1,
$$

where $C_{\mathrm{pm}}$ is the cost of a single PM, $C_{r}$ is the cost of a single $\mathrm{RM}$, and $C_{\mathrm{pm}} \leq C_{r} ; \eta$ is the cost adjustment factor, and $1 \leq \eta \leq\left(C_{r} / C_{\mathrm{pm}}\right) ; q$ is the maintenance time adjustment factor, and $q>0 ;(i+c)^{v}$ is the Stanford-B learning curve, and $c$ is the experience parameter, $1<c<10$. A larger $c$ indicates a higher experience value, $v$ is an adjustment factor for the maintenance learning effect, $v=(\ln \varphi / \ln 2), \varphi$ is the experience curve percentage, and $z$ is the PM parameter.

Assume that each PM of the equipment cannot be repaired as new but returns to the previous state, and the amount of return is $\gamma_{i} \tau_{i}$. The next time the system runs, its state does not start from 0 but from a state $\psi_{i}$ after $0 . \psi_{i}$ represents the equipment recovery after the $i$-th PM. The equipment virtual age after the $i$-th PM is

$$
\left\{\begin{array}{l}
\psi_{1}=\tau_{1}-\gamma_{1} \tau_{1} \\
\psi_{2}=\psi_{1}+\tau_{2}-\gamma_{2} \tau_{2} \\
\cdots \\
\psi_{n}=\psi_{n-1}+\tau_{n}-\gamma_{n} \tau_{n}=\sum_{i=1}^{n}\left(1-\gamma_{i}\right) \tau_{i}
\end{array}\right.
$$

Assume that the equipment failure rate function is $\lambda(t)$; the failure rate model considering the virtual age reduction factor after the $i$-th PM is

$$
\left\{\begin{array}{l}
\lambda_{1}(t)=\lambda(t) \\
\lambda_{2}(t)=\lambda_{1}\left(t+\tau_{1}-\gamma_{1} \tau_{1}\right) \\
\cdots \\
\lambda_{n}(t)=\lambda_{n-1}\left(t+\tau_{n-1}-\gamma_{n-1} \tau_{n-1}\right)=\lambda\left(t+\sum_{j=1}^{i}\left(1-\gamma_{j}\right) \tau_{j}\right), t \in\left[0, \tau_{i+1}\right)
\end{array}\right.
$$

The incremental failure rate factor means that the failure rate function slope will increase after each PM. Reflected in the equipment failure, it means that the equipment will enter the next PM at a faster speed. In this paper, $\mu_{i}$ is used to represent the incremental failure rate factor of the $i$-th PM.
After each PM, the failure rate function will change to a certain degree, and its expression is as follows:

$$
\mu_{i}=\frac{f_{1} i+g_{1}}{f_{2} i+g_{2}}, \mu_{i} \geq 1,
$$




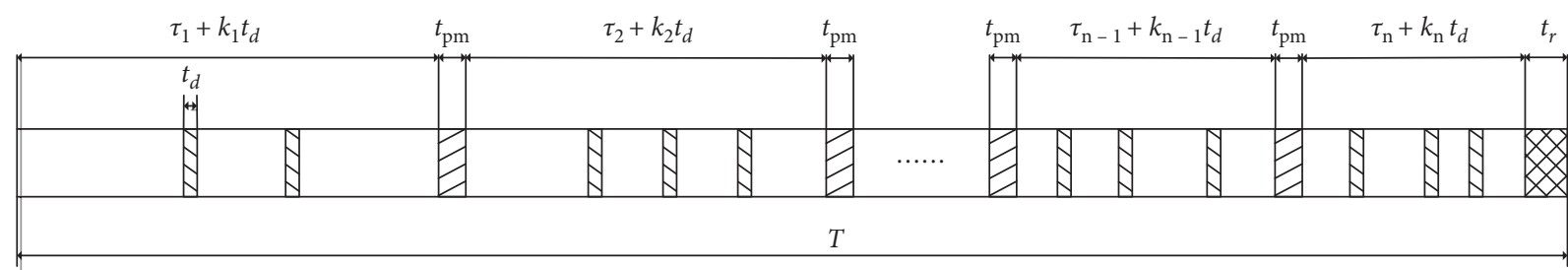

BM time $甘$ PM time RM time

Figure 1: Combined maintenance strategy.

where $f_{1}, f_{2}, g_{1}$, and $g_{2}$ are the adjustment factors of the incremental failure rate.

The failure rate model considering the incremental failure rate factor after the $i$-th PM is

$$
\left\{\begin{array}{l}
\lambda_{1}(t)=\lambda(t) \\
\lambda_{2}(t)=\mu_{1} \lambda_{1}(t) \\
\lambda_{3}(t)=\mu_{2} \lambda_{2}(t), \\
\cdots \\
\lambda_{n}(t)=\mu_{n-1} \lambda_{n-1}(t)=\lambda\left(t+\sum_{j=1}^{i}\left(1-\gamma_{j}\right) \tau_{j}\right), t \in\left[0, \tau_{i+1}\right) .
\end{array}\right.
$$

This paper combines the two failure rate models to establish a mixed failure rate model based on two factors, the virtual age reduction factor and the incremental failure rate factor, making it a more realistic and reasonable model for the equipment. The mixed failure rate function is generally distributed as

$$
\lambda_{i+1}(t)=\left(\prod_{j=1}^{i} \mu_{j}\right) \lambda\left(t+\sum_{j=1}^{i}\left(1-\gamma_{j}\right) \tau_{j}\right), t \in\left[0, \tau_{i+1}\right) .
$$

The Weibull distribution is widely used in reliability engineering, especially suitable for the distribution form of cumulative wear and tear of mechanical and electrical products. Assume that the production line equipment failure obeys the two-parameter Weibull distribution [32], and its failure rate and reliability function are, respectively, expressed in the two following equations:

$$
\begin{aligned}
& \lambda(t)=\frac{\beta}{\alpha}\left(\frac{t}{\alpha}\right)^{\beta-1}, \quad t \geq 0, \\
& R(t)=\exp \left[-\left(\frac{t}{\alpha}\right)^{\beta}\right], \quad t \geq 0,
\end{aligned}
$$

where $\beta$ is the shape parameter and $\alpha$ is the scale parameter.

Combining equations (28) and (29), the number of equipment failures in each PM cycle is expressed in the following equation:

$$
\begin{aligned}
k_{i} & =\int_{\psi_{i-1}}^{\tau_{i}+\psi_{i-1}} \lambda_{i+1}(t) \mathrm{d} t \\
& =\int_{\psi_{i-1}}^{\tau_{i}+\psi_{i-1}}\left(\prod_{j=1}^{i} \mu_{j}\right) \frac{\beta}{\alpha}\left(\frac{t}{\alpha}\right)^{\beta-1} \mathrm{~d} t \\
& =\left(\prod_{i=1}^{n} \mu_{i}\right)\left[\left(\frac{\tau_{i}+\psi_{i-1}}{\alpha}\right)^{\beta}-\left(\frac{\psi_{i-1}}{\alpha}\right)^{\beta}\right] \\
& =\left(\prod_{i=1}^{n} \mu_{i}\right)\left[\left(\frac{\tau_{i}+\sum_{i=1}^{n-1}\left(1-\gamma_{i}\right) \tau_{i}}{\alpha}\right)^{\beta}-\left(\frac{\sum_{i=1}^{n-1}\left(1-\gamma_{i}\right) \tau_{i}}{\alpha}\right)^{\beta}\right] .
\end{aligned}
$$

\subsection{PM Model for Production Line Important Equipment} Based on the Mixed Failure Rate. Important equipment has a great influence on the production line. Once the equipment fails, it will have a greater impact on the entire line. Therefore, the downtime should be shortened as far as possible and the availability and reliability of the equipment should be improved. When the stability of important equipment is required, the equipment availability will be given priority when adopting a PM strategy. Availability can comprehensively measure the reliability and maintainability of the important equipment. Therefore, this paper establishes a PM model with the reliability as the constraint and the maximum availability as the optimization objective for important equipment.

Availability refers to the probability that the equipment is in a workable or usable state under specified conditions and time. It can be expressed as the ratio of normal working time to total time.

$$
A(t)=\frac{T_{w}}{T_{w}+T_{d}},
$$

where $T_{w}$ is the normal working time and $T_{d}$ is the downtime of the equipment.

Considering the influence of the mixed failure rate, the equipment availability expression in the $i$-th PM cycle is 


$$
A\left(\tau_{i}\right)= \begin{cases}\frac{\tau_{i}}{\tau_{i}+k_{i} t_{d}+t_{\mathrm{pm}}}, & i=1,2, \ldots, n-1, \\ \frac{\tau_{i}}{\tau_{i}+k_{i} t_{d}+t_{r}}, & i=n .\end{cases}
$$

The equipment availability in a preventive maintenance replacement cycle is

$$
A\left(n, \tau_{1}, \tau_{2}, \ldots, \tau_{n}\right)=\frac{\sum_{i=1}^{n} \tau_{i}}{\sum_{i=1}^{n}\left(\tau_{i}+k_{i} t_{d}\right)+(n-1) t_{\mathrm{pm}}+t_{r}} .
$$

From equations (29) and (30), it can be known that there is a certain relationship between the equipment reliability and the failure rate function:

$$
R(t)=\exp \left(-\int_{0}^{t} \lambda(t) \mathrm{d} t\right)
$$

After each PM, the failure rate function is constantly changing, so equation (35) cannot be directly used, but the reliability calculation should be performed in sections. The equipment reliability in the $i$-th maintenance cycle is

$$
R\left(\tau_{i}\right)=\exp \left(-\int_{0}^{\tau_{i}} \lambda_{i}(t) \mathrm{d} t\right) .
$$

Take the logarithm of both sides of equation (36) at the same time:

$$
\int_{0}^{\tau_{i}} \lambda_{i}(t) \mathrm{d} t=-\ln R\left(\tau_{i}\right),
$$

where $\int_{0}^{\tau_{i}} \lambda_{i}(t) \mathrm{d} t$ is the number of equipment failures in the $i$-th PM cycle. According to equation (37), when the constraint condition is reliable, the number of equipment failures in each PM cycle is $-\ln R$.

This paper establishes an important equipment PM model with the maximum availability as the optimization objective, and the reliability threshold value is $R_{0}$; each PM process must ensure that $R\left(\tau_{i}\right)>R_{0}$. The important equipment PM model based on availability is

$$
\begin{aligned}
& \max A\left(n, \tau_{1}, \tau_{2}, \ldots, \tau_{n}\right)=\frac{\sum_{i=1}^{n} \tau_{i}}{\sum_{i=1}^{n}\left(\tau_{i}+k_{i} t_{d}\right)+(n-1) t_{\mathrm{pm}}+t_{r}} \\
& \text { s.t. } \exp \left(-\int_{0}^{\tau_{i}} \lambda_{i}(t) \mathrm{d} t\right) \geq R_{0} \\
& \sum_{i=1}^{n}\left(\tau_{i}+k_{i} t_{d}\right)+(n-1) t_{\mathrm{pm}}+t_{r} \leq T
\end{aligned}
$$$$
\tau_{i} \geq k_{i} t_{d},
$$

where the optimal number of PM $n$ and the PM cycle $\tau_{i}$ are decision variables.
4.3. PM Model for Production Line Secondary Equipment Based on the Mixed Failure Rate. The influence of the secondary equipment on the production line is lower than that of the important equipment. Enterprises pay more attention to the problem of saving maintenance costs, but blindly reducing costs may cause frequent equipment failures. In order to solve the above problems, under the limitation of the PM reliability threshold value $R_{0}$, this paper establishes a secondary equipment PM model with the minimum PM cost rate as the optimization objective.

The maintenance cost is closely related to the maintenance method. The secondary equipment adopts the combined maintenance strategy of multiple $\mathrm{BM}, n-1 \mathrm{PM}$, and $n-$ th RM in one equipment maintenance replacement cycle. Therefore, the total equipment maintenance costs are composed of three parts: (1) The BM cost includes the minor maintenance $\operatorname{cost} C_{\mathrm{mr}}$, downtime $\operatorname{cost} C_{d}$, fixed $\operatorname{cost} C_{f}$, and penalty $\operatorname{cost} C_{p}$. The fixed cost includes the cost of each test and so forth. The penalty cost refers to the extra cost incurred by investing more maintenance resources when the maintenance time exceeds the specified time limit. (2) The $\mathrm{PM}$ cost includes the maintenance cost $C_{\mathrm{pm}}$, downtime cost $C_{d}$, and fixed $\operatorname{cost} C_{f}$. (3) At the end of the $n$-th PM cycle, the equipment RM cost includes the maintenance cost $C_{r}$, downtime cost $C_{d}$, and fixed cost $C_{f}$.

The total BM cost is

$$
C_{\mathrm{tm}}=\left(C_{\mathrm{mr}}+t_{\mathrm{mr}} C_{d}+C_{f}+\delta C_{p}\right) \sum_{i=1}^{n} k_{i},
$$

where $t_{\mathrm{mr}}$ is the average maintenance time of $\mathrm{BM}$ and $\delta$ is the probability of maintenance overtime.

The total PM cost is

$$
C_{\mathrm{tp}}=\left(C_{\mathrm{pm}}+t_{\mathrm{pm}} C_{d}+C_{f}\right)(n-1),
$$

where $t_{\mathrm{pm}}$ is the average maintenance time of PM.

The total RM cost is

$$
C_{t r}=C_{r}+t_{r} C_{d}+C_{f}
$$

where $t_{r}$ is the average duration time of RM.

The total cost of a preventive maintenance replacement cycle of the equipment is

$$
C=C_{\mathrm{tm}}+C_{\mathrm{tp}}+C_{\mathrm{tr}}
$$

A preventive maintenance replacement cycle $T$ is

$$
T=\sum_{i=1}^{n}\left(\tau_{c i}+k_{i} t_{\mathrm{mr}}\right)+(n-1) t_{\mathrm{pm}}+t_{r}
$$

The maintenance cost rate of a preventive maintenance replacement cycle is 


$$
\begin{aligned}
C\left(n, \tau_{1}, \tau_{2}, \ldots, \tau_{n}\right) & =\frac{C}{T} \\
& =\frac{\left(C_{\mathrm{mr}}+t_{\mathrm{mr}} C_{d}+C_{f}+h C_{p}\right) \sum_{i=1}^{n} k_{i}+\left(C_{\mathrm{pm}}+t_{\mathrm{pm}} C_{d}+C_{f}\right)(n-1)+\left(C_{r}+t_{r} C_{d}+C_{f}\right)}{\sum_{i=1}^{n}\left(\tau_{i}+k_{i} t_{\mathrm{mr}}\right)+(n-1) t_{\mathrm{pm}}+t_{r}} .
\end{aligned}
$$

This paper establishes a secondary equipment PM model with the minimum maintenance cost rate as the optimization objective, and the reliability threshold value is $R_{0}$.
From equations (36) and (44), the secondary equipment PM model based on the maintenance cost rate can be obtained.

$$
\begin{aligned}
& \min C\left(n, \tau_{1}, \tau_{2}, \ldots, \tau_{n}\right) \\
& =\frac{\left(C_{\mathrm{mr}}+t_{\mathrm{mr}} C_{d}+C_{f}+h C_{p}\right) \sum_{i=1}^{n} k_{i}+\left(C_{\mathrm{pm}}+t_{\mathrm{pm}} C_{d}+C_{f}\right)(n-1)+\left(C_{r}+t_{r} C_{d}+C_{f}\right)}{\sum_{i=1}^{n}\left(\tau_{i}+k_{i} t_{\mathrm{mr}}\right)+(n-1) t_{\mathrm{pm}}+t_{r}}, \\
& \text { s.t. } \exp \left(-\int_{0}^{\tau_{i}} \lambda_{i}(t) \mathrm{d} t\right) \geq R_{0} \\
& \sum_{i=1}^{n}\left(\tau_{i}+k_{i} t_{\mathrm{mr}}\right)+(n-1) t_{\mathrm{pm}}+t_{r} \leq T \tau_{i} \geq k_{i} t_{\mathrm{mr}}
\end{aligned}
$$

where the optimal number of PM $n$ and the PM cycle $\tau_{i}$ are decision variables.

\section{Example Application of Production Line Equipment}

A production line consists of a series of processing machine tools with equipment numbers $m_{1}-m_{20}$. The layout is shown in Figure 2.

5.1. Division of Production Line Equipment. The grey interval AHP-Entropy method is used to divide the production line equipment. According to the equipment importance evaluation index, a judgment matrix is constructed based on the evaluation opinions of five experts to determine comprehensive weight of the index, as shown in Table 1.

The evaluation criteria are divided into five levels \{higher, high, medium, low, and lower\} and are assigned values of $9,7,5,3$, and 1 , respectively, according to the equipment importance. When the importance is higher than the maximum value, between two adjacent importance degrees, or lower than the minimum value, the values assigned are $10,8,6,4,2$, and 0 , respectively. Organize 5 experts $(k=5)$ to score 20 pieces of evaluated equipment $(s=20)$ according to the evaluation index $U_{i}$ and organize the data information into a matrix form. Record the evaluation sample matrix of the first evaluated equipment as $D^{(1)}$ as follows:

$$
D^{(1)}=\left[\begin{array}{lllll}
d_{11}^{(1)} & d_{12}^{(1)} & d_{13}^{(1)} & d_{14}^{(1)} & d_{15}^{(1)} \\
d_{21}^{(1)} & d_{22}^{(1)} & d_{23}^{(1)} & d_{24}^{(1)} & d_{25}^{(1)} \\
d_{31}^{(1)} & d_{32}^{(1)} & d_{33}^{(1)} & d_{34}^{(1)} & d_{35}^{(1)} \\
d_{41}^{(1)} & d_{42}^{(1)} & d_{43}^{(1)} & d_{44}^{(1)} & d_{45}^{(1)} \\
d_{51}^{(1)} & d_{52}^{(1)} & d_{53}^{(1)} & d_{54}^{(1)} & d_{55}^{(1)}
\end{array}\right]=\left[\begin{array}{lllll}
3 & 5 & 3 & 2 & 1 \\
5 & 3 & 3 & 1 & 4 \\
2 & 3 & 5 & 5 & 2 \\
4 & 2 & 2 & 1 & 2 \\
3 & 4 & 3 & 2 & 1
\end{array}\right] .
$$

This paper takes the grey evaluation as four levels of excellent, good, medium, and poor; that is, $e=1,2,3,4$. The corresponding grey category, whitening weight function expressions, and diagrams are shown in Table 2.

For the evaluation index $U_{i}$, the grey evaluation coefficient $\sigma_{i e}^{(1)}$ of the first equipment belonging to the $e$-th grey category and the total grey evaluation coefficient $\sigma_{i}^{(1)}$ belonging to each grey category can be calculated by formulas (20) and (21). The grey evaluation weight $r_{i e}^{(1)}$ of the first equipment and the grey evaluation weight vector $r_{i}^{(1)}$ for each grey category can be calculated by formulas (19) and (20). Therefore, the grey evaluation weight matrix $R^{(1)}$ of the first equipment for each grey category is obtained as

$$
R^{(1)}=\left[\begin{array}{l}
r_{1}^{(1)} \\
r_{2}^{(1)} \\
r_{3}^{(1)} \\
r_{4}^{(1)} \\
r_{5}^{(1)}
\end{array}\right]=\left[\begin{array}{cccc}
0.3578 & 0.3670 & 0.1651 & 0.1101 \\
0.4369 & 0.3884 & 0.0583 & 0.1165 \\
0.4286 & 0.3429 & 0.2286 & 0 \\
0.2683 & 0.2927 & 0.3415 & 0.0976 \\
0.3451 & 0.3894 & 0.1593 & 0.1062
\end{array}\right] .
$$




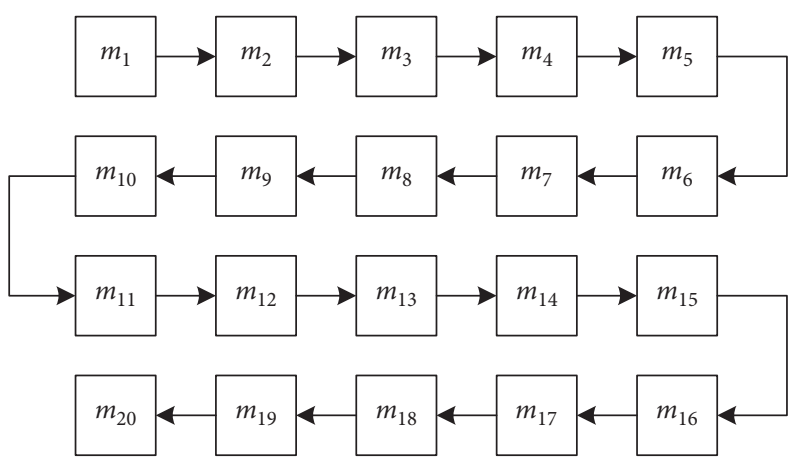

FIgURE 2: Production line equipment layout.

TABLE 1: Judgment matrix on the importance evaluation $U$ of production line equipment.

\begin{tabular}{ccccccc}
\hline & \multicolumn{3}{c}{ Evaluation index } & & $U_{5}$ & Combined weight \\
$U$ & $U_{1}$ & $U_{2}$ & $U_{3}$ & $U_{4}$ & 0.2838 \\
\hline$U_{1}$ & {$[1,1]$} & {$[3,4]$} & {$[4,11 / 2]$} & {$[2,7 / 2]$} & {$[5,7]$} & 0.1908 \\
$U_{2}$ & {$[1 / 4,1 / 3]$} & {$[1,1]$} & {$[5 / 2,9 / 2]$} & {$[1 / 2,1]$} & {$[1 / 4,1 / 3]$} & 0.2217 \\
$U_{3}$ & {$[2 / 11,1 / 4]$} & {$[2 / 9,2 / 5]$} & {$[1,1]$} & {$[1 / 5,1]$} & {$[5,7]$} & 0.1516 \\
$U_{4}$ & {$[2 / 7,1 / 2]$} & {$[1,2]$} & {$[1,5]$} & {$[1,1]$} & {$[3,5]$} & 0.1521 \\
$U_{5}$ & {$[1 / 7,1 / 5]$} & {$[3,4]$} & {$[1 / 7,1 / 5]$} & {$[1 / 5,1 / 3]$} & {$[1,1]$} & \\
\hline
\end{tabular}

A comprehensive evaluation of $U$ of the first equipment is made by formula (21), and the result is $C^{(1)}=W \cdot R^{(1)}=(0.3731,0.3579,0.1846,0.0844)$, and assume that $E=\left(d_{1}, d_{2}, d_{3}, d_{4}\right)=(8,6,4,2)$; the comprehensive evaluation value $Z^{(1)}$ of the first evaluated equipment is $Z^{(1)}=C^{(1)} \cdot E^{(T)}=6.0392$.

Using the same method to calculate the comprehensive evaluation value of the remaining evaluated equipment, the equipment is sorted according to the size of the comprehensive evaluation value. Finally use the ABC classification method to divide the importance of the equipment. Consider the top $20 \%$ of equipment as the important equipment, the $20 \%-50 \%$ of equipment as the secondary equipment, and the remaining $50 \%$ of equipment as the general equipment. According to the calculation results, the equipment numbered $3,5,8$, and 12 is defined as the important equipment, the equipment numbered $4,6,9,13,15$, and 17 is defined as the secondary equipment, and the remaining is defined as the general equipment.

5.2. Maintenance Strategy of Production Line Equipment. For important equipment and secondary equipment, a combined maintenance method is adopted. Two PM models are established with constraints of reliability and optimization objective of maximum availability and minimum maintenance cost rate. Assume that the failure rate distribution of important and secondary equipment obeys the two-parameter Weibull distribution of $\beta=2.3378$ and $\alpha=6179.2932$ as well as $\beta=1.6074$ and $\alpha=6315.8233$, respectively. Other related parameters are shown in Table 3.

Consider the equipment reliability threshold value $\mathrm{R}_{0} \in[0.75,0.95]$. Matlab simulation is used to solve the important equipment PM model based on availability. The relationship curves $\left(R_{0}, n\right)$ between the reliability threshold value and the optimal number of PM and the maximum availability and PM cycle under $\left(R_{0}, n\right)$ are obtained. The availability change with the number of PM is shown in Figure 3.

It can be known from Figure 3 that, under the constraint of the reliability threshold value, the availability decreases with an increase of the number of PM. When the number of PM $n<5$, the availability increases with the number of PM. When the number of PM $n>5$, the availability decreases with the number of PM. Therefore, when the number of PM $n=5$, there is an optimal value to make the highest equipment availability under the PM strategy. When the number of PM $n$ is constant, the equipment availability decreases with the increase of the reliability threshold value $R_{0}$. This is because the higher $R_{0}$ is, the shorter the overall equipment PM replacement cycle is, which will shorten the normal operation time of the equipment, resulting in decrease of equipment availability.

Similarly, assume that the equipment reliability threshold value $R_{0} \in[0.75,0.95]$. Matlab simulation is used to solve the secondary equipment PM model based on maintenance cost rate and the relationship curves $\left(R_{0}, n\right)$ between the reliability threshold value and the optimal number of $P M$, and the minimum maintenance cost rate and PM cycle under $\left(R_{0}, n\right)$ are obtained. The change of maintenance cost rate with the number of PM is shown in Figure 4.

Taking $R_{0}=0.95, n=10,11, \ldots, 15$ as an example, the partial enlarged diagram of the maintenance cost rate change with the number of PM is shown in the upper right corner of Figure 4.

From Figure 4, we know that, along with the increase of the number of $\mathrm{PM}$, the equipment maintenance cost rate will 
TABLE 2: Equipment importance evaluation whitening weight function and diagram.

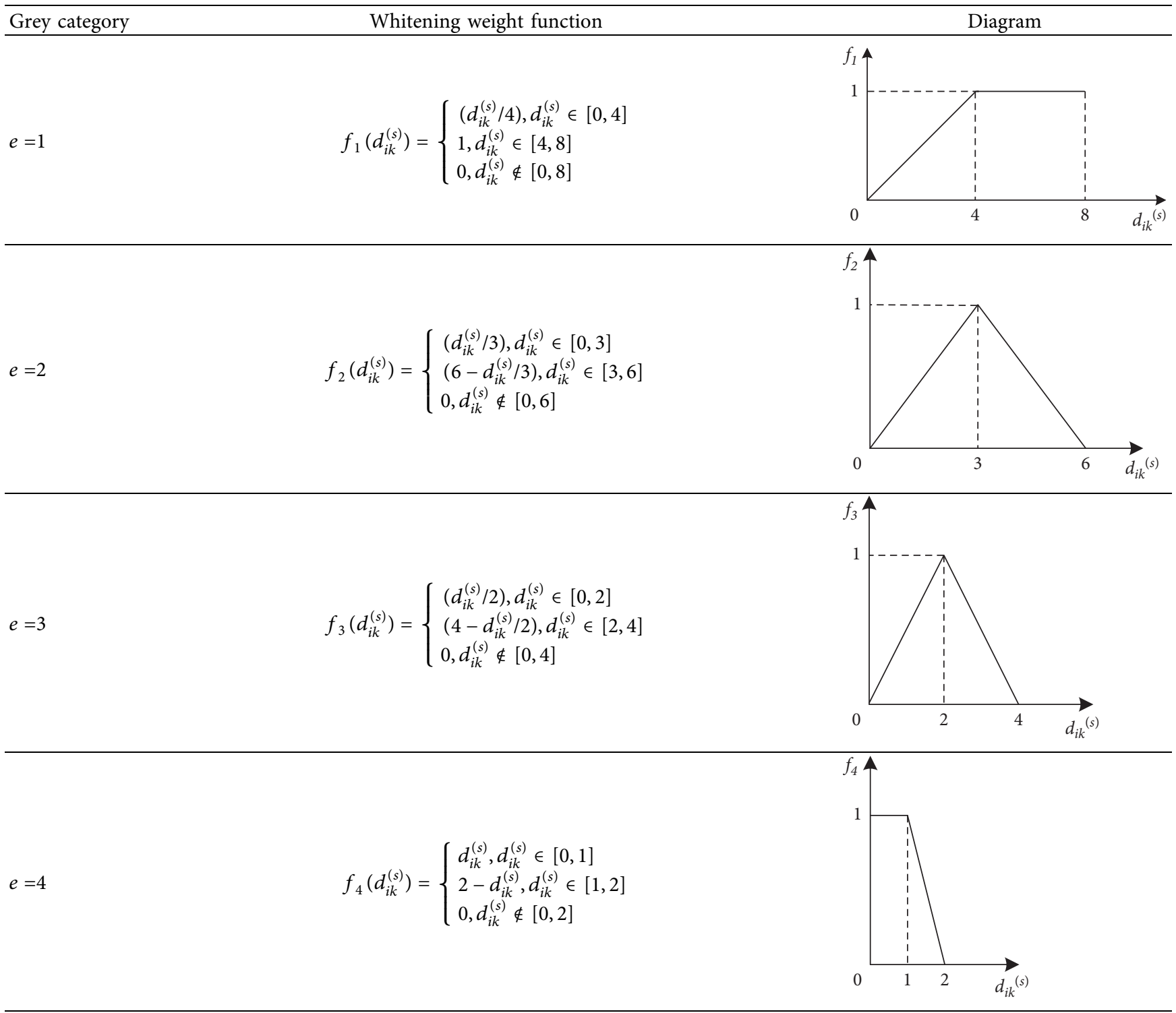

TABLE 3: Related parameters of production line equipment.

\begin{tabular}{lcc}
\hline Parameter & Important equipment & Secondary equipment \\
\hline BM cost (yuan) & 1200 & 800 \\
Downtime cost (yuan) & 300 & 100 \\
Fixed cost (yuan) & 1200 & 800 \\
Penalty cost (yuan) & 2000 & 1000 \\
PM cost (yuan) & 4000 & 2000 \\
RM cost (yuan) & 50000 & 30000 \\
BM time (hour) & 5 & 3 \\
PM time (hour) & 15 & 10 \\
RM time (hour) & 24 & 20 \\
Cost adjustment factor & 1 & 1 \\
Maintenance time adjustment factor & 0.002 & 0.002 \\
Experience parameter & 4 & 4 \\
Experience curve percentage & 0.8 & 0.8 \\
PM parameter & 0.02 & 0.02 \\
Adjustment factor $f_{1}$ & 12 & 12 \\
Adjustment factor $f_{2}$ & 11 & 11 \\
Adjustment factor $g_{1}$ & 1 & 1 \\
Adjustment factor $g_{2}$ & 1 & 1 \\
Probability of maintenance overtime & 0.15 & 0.15 \\
\hline
\end{tabular}




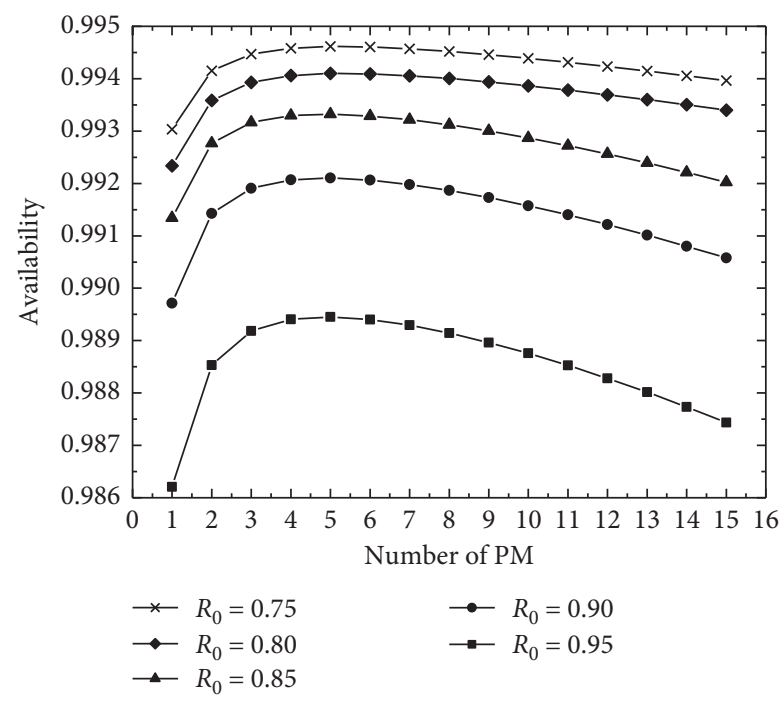

FIGURE 3: Equipment availability model.

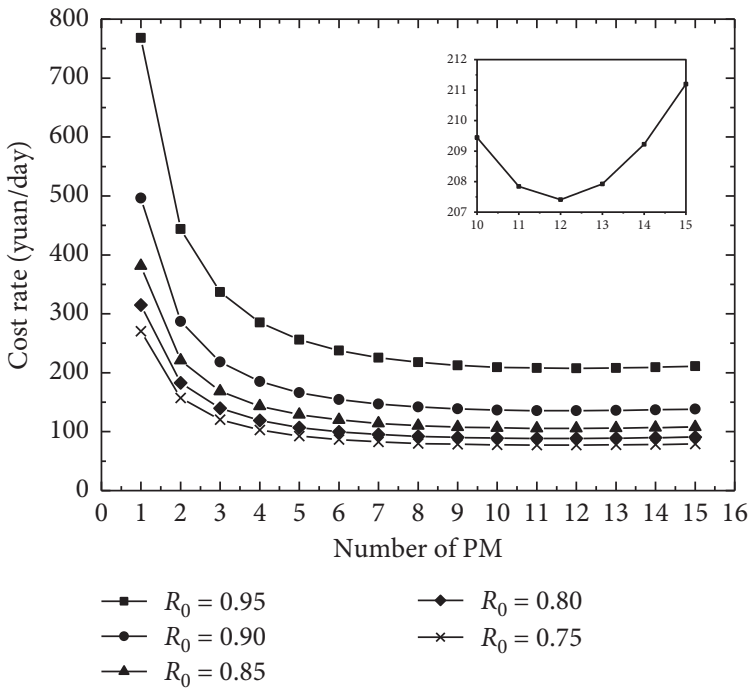

FIgURE 4: Equipment maintenance cost rate model.

decrease first and then increase under the constraint of reliability threshold value $R_{0}$. There must be a minimum equipment maintenance cost rate under the PM strategy, and the number of PM $n$ is the minimum point of it. The optimal number of PM for the reliability threshold $R_{0}=0.75$ is 11 times, and the optimal number of PM for $R_{0}=0.80$, $0.85,0.90$, and 0.95 is 12 times. The higher the reliability threshold value, the higher the optimal number of PM and the maintenance cost rate. When the number of PM is constant, the equipment maintenance cost rate increases along with the increase of the reliability threshold value. Because the higher the reliability threshold value, the more costs of each PM investment and the shorter the overall equipment preventive maintenance replacement cycle, this increases the equipment maintenance cost rate.

Tables 4 and 5 show the PM cycle $\tau_{i}$, availability $A$, and maintenance cost rate $C$ of each PM cycle corresponding to the optimal maintenance strategy under the constraints of each reliability threshold value $R_{0}$.

From Tables 4 and 5, we know that, in order to meet the requirements of the reliability threshold value, the PM cycle $\tau_{i}$ will be gradually shortened, and the overall equipment preventive maintenance replacement cycle decreases along with the reliability threshold value increases. The virtual age reduction factor and the incremental failure rate factor in this paper are dynamic. With the number of PM increasing, the virtual age reduction factor gradually decreases and the incremental failure rate factor gradually increases, which makes the equipment age recovery capability after PM constantly weakened, and the degree of aging wear and tear gradually increased.

The important equipment has a great impact on the production line. It is necessary to shorten its downtime and improve reliability as much as possible. The optimal 
TABLE 4: Combined maintenance strategy for important equipment.

\begin{tabular}{lcc}
\hline$\left(R_{0}, n\right)$ & PM cycle (day) & Availability \\
\hline$(0.75,5)$ & $\tau_{1}=151.10, \tau_{2}=145.56, \tau_{3}=140.18, \tau_{4}=134.95, \tau_{5}=129.89$ & 0.9946 \\
$(0.80,5)$ & $\tau_{1}=135.54, \tau_{2}=130.57, \tau_{3}=125.22, \tau_{4}=121.06, \tau_{5}=116.52$ & 0.9941 \\
$(0.85,5)$ & $\tau_{1}=118.36, \tau_{2}=114.01, \tau_{3}=109.34, \tau_{4}=104.51, \tau_{5}=99.59$ & 0.9933 \\
$(0.90,5)$ & $\tau_{1}=98.33, \tau_{2}=94.72, \tau_{3}=90.84, \tau_{4}=86.83, \tau_{5}=82.74$ & 0.9921 \\
$(0.95,5)$ & $\tau_{1}=72.27, \tau_{2}=69.62, \tau_{3}=66.77, \tau_{4}=63.82, \tau_{5}=60.81$ & 0.9895 \\
\hline
\end{tabular}

TABLE 5: Combined maintenance strategy for secondary equipment.

\begin{tabular}{|c|c|c|}
\hline$\left(R_{0}, n\right)$ & PM cycle (day) & $\begin{array}{l}\text { Cost rate (yuan/ } \\
\text { day) }\end{array}$ \\
\hline$(0.75,11)$ & $\begin{array}{c}\tau_{1}=121.23, \tau_{2}=114.97, \tau_{3}=108.50, \tau_{4}=102.04, \tau_{5}=95.66, \tau_{6}=89.42, \tau_{7}=83.35, \tau_{8}=77.46 \\
\tau_{9}=71.76, \tau_{10}=66.27, \tau_{11}=60.98\end{array}$ & 77.1168 \\
\hline$(0.80,12)$ & $\begin{aligned} \tau_{1}=103.50, \tau_{2}=98.17, \tau_{3}=92.64, \tau_{4}=87.12, \tau_{5} & =81.68, \tau_{6}=76.35, \tau_{7}=71.16, \tau_{8}=66.13, \tau_{9}=61.27, \\
\tau_{10}=56.58, \tau_{11} & =52.06, \tau_{12}=47.73\end{aligned}$ & 88.5288 \\
\hline$(0.85,12)$ & $\begin{aligned} \tau_{1}=84.98, \tau_{2}=80.60, \tau_{3}=76.06, \tau_{4} & =71.53, \tau_{5}=67.06, \tau_{6}=62.68, \tau_{7}=58.43, \tau_{8}=54.30, \tau_{9}=50.30 \\
\tau_{10} & =46.45, \tau_{11}=42.75, \tau_{12}=39.19\end{aligned}$ & 105.6768 \\
\hline$(0.90,12)$ & $\begin{aligned} \tau_{1}=64.89, \tau_{2}=61.55, \tau_{3}=58.08, \tau_{4} & =54.62, \tau_{5}=51.21, \tau_{6}=47.87, \tau_{7}=44.62, \tau_{8}=41.46, \tau_{9}=38.41 \\
\tau_{10} & =35.47, \tau_{11}=32.64, \tau_{12}=29.93\end{aligned}$ & 135.5952 \\
\hline$(0.95,12)$ & $\begin{aligned} \tau_{1}=41.47, \tau_{2}=39.33, \tau_{3}=37.12, \tau_{4} & =34.90, \tau_{5}=32.72, \tau_{6}=30.59, \tau_{7}=28.51, \tau_{8}=26.50, \tau_{9}=24.55 \\
\tau_{10} & =22.67, \tau_{11}=20.86, \tau_{12}=19.12\end{aligned}$ & 207.4080 \\
\hline
\end{tabular}

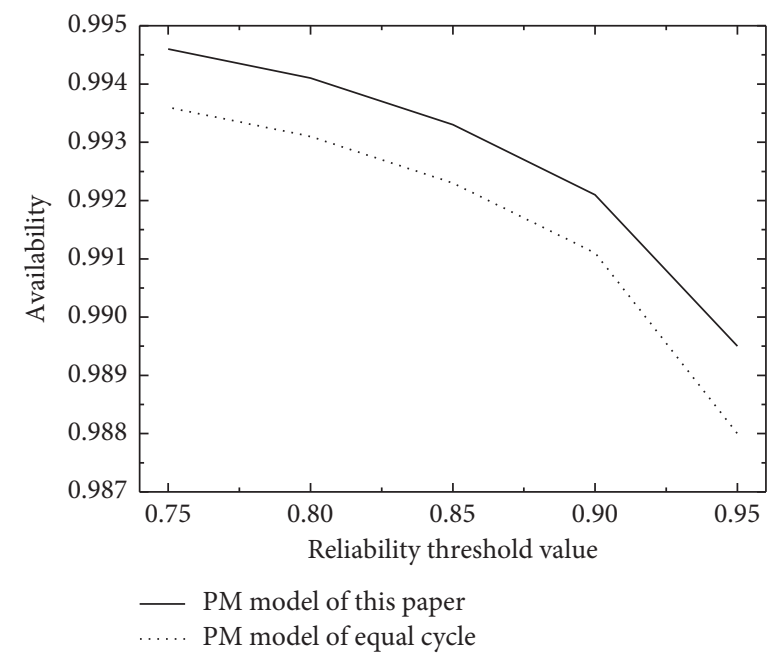

Figure 5: Comparison of the availability.

combined maintenance strategy for the important equipment is $(0.95,5)$. BM and PM are adopted before the fifth time and RM is adopted on the fifth time. Under the premise of ensuring the reliability requirement, enterprises pay more attention to the problem of saving maintenance costs for the secondary equipment, and the optimal combined maintenance strategy is $(0.80,12)$. BM and PM are adopted before the 12th time and RM is adopted on the 12th time. The maintenance strategies for important equipment and secondary equipment play a vital role in improving the equipment reliability, reducing maintenance costs, and reducing equipment failures due to the unreasonable maintenance schedule.

In order to further verify the effectiveness of the model proposed in this paper, the model is compared with the traditional equal cycle PM model. Taking the optimal maintenance strategy $(0.80,12)$ of the secondary equipment as an example, the equal cycle PM model means that there are 11 equal PM cycles in a preventive maintenance replacement cycle, and the equipment will be replaced during the 12th PM. For the important equipment and secondary equipment of the production line, under the same PM number and reliability constraints, the same Weibull distribution parameters and other parameters in Table 3 are substituted into the equal cycle PM model. The availability and cost rate corresponding to the reliability threshold are obtained, respectively, as shown in Figures 5 and 6.

It can be seen from Figure 5 that, under the same PM number and reliability threshold, both the PM model proposed in this paper and the equal cycle model show that 


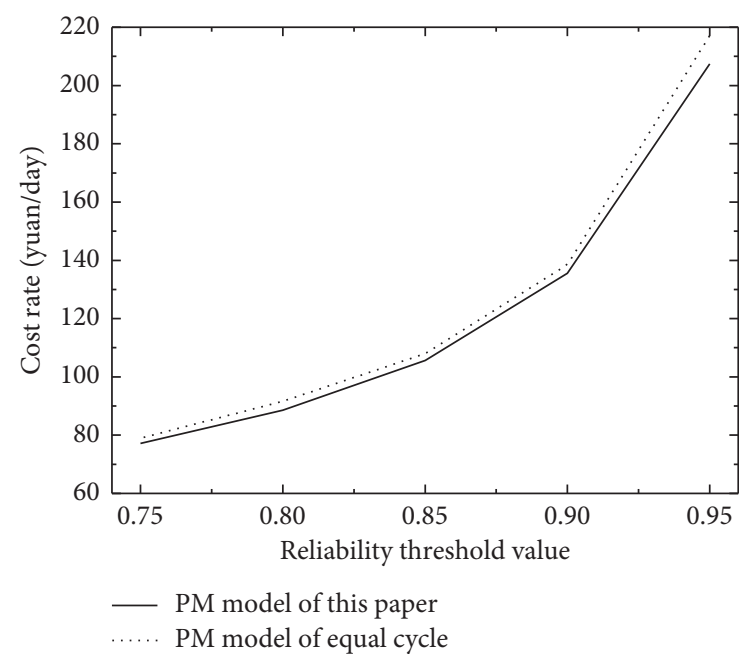

Figure 6: Comparison of the maintenance cost rate.

the availability decreases with the increase of the reliability threshold value, but the difference between them is that the availability of the equal cycle model is always lower than that of the PM model established in this paper. It can be seen from Figure 6 that the maintenance cost rate of the model proposed in this paper and the equal cycle model both increase with the increase of the reliability threshold value, but the cost rate of the equal cycle model is always higher than that of the model established in this paper. Therefore, the PM model in this paper ensures the high reliability of the equipment and effectively reduces the maintenance cost.

\section{Conclusion}

(1) In this paper, a comprehensive evaluation method combining interval AHP-Entropy method and grey evaluation method is used to divide the production line equipment. When determining the weight of equipment importance, a comprehensive weighting method is used, which combines interval numbers with AHP and Entropy method, which not only makes the index evaluation reach the unity of subjectivity and objectiveness but also effectively solves the problem of uncertain evaluation. It is a more scientific, comprehensive, and reliable method for equipment division.

(2) A dynamic virtual age reduction factor related to PM costs, RM costs, equipment age, the number of maintenances, maintenance learning effect, and personnel experience factors and an incremental failure rate factor related to the number of PM are introduced to establish the mixed failure rate model. The model describes the change in the failure rate of the equipment more scientifically and realistically during the PM cycle.

(3) For the important equipment and secondary equipment of the production line, a combined maintenance method combining BM, PM, and RM is adopted. Two PM models are established with the

constraint of reliability and optimization objective of maximum availability and minimum maintenance cost rate to determine the optimal PM times and cycles. Combined with an example of production line equipment, the optimal combined maintenance strategies for important equipment and secondary equipment are obtained, respectively, $(0.95,5)$ and $(0.80,12)$ and are then compared with the PM model of equal cycle. The results verified the feasibility of the model, which effectively improve the production line equipment reliability and reduce maintenance costs.

\section{Notations}

$U_{i}:$

$w_{\mathrm{AHP}}$ and

$w_{\mathrm{EM}}:$

$w_{i}$ :

$a_{i j}$ and $b_{i j}$ :

$g_{i j}$ and $h_{i j}$ :

$x_{i}$ and $y_{i}$ :

$s:$

$d_{i k}^{(s)}:$

$e$ :

$f_{e}\left(d_{i k}^{(s)}\right)$

$\sigma_{i e}^{(s)}:$

$\sigma_{i}^{(s)}$ :

$r_{i e}^{(s)}$ :

$r_{i}^{(s)}$ :

$R^{(s)}$ :

$C^{(s)}$ :

$Z^{(s)}$ :

$T$ :

$n:$

$\tau_{i}:$

$k_{i}$ :

$\gamma_{i}$ :
The $i$-th evaluation index

The weight interval ranges of the interval AHP and interval Entropy method The combined weight of the $i$-th evaluation index

The upper and lower limits of the judgment result, $i, j=1,2, \cdots, m$

The proportion of the $j$-th value in the lower and upper limits under the $i$-th index

The information Entropy values of the lower and upper limits of the $i$-th index

The number of the evaluated pieces of equipment

The evaluation result of the $k$-th expert on the $s$-th evaluated equipment according to the evaluation index $U_{i}$

The grey category in the grey evaluation method, $e=1,2, \cdots, p$

The whitening weight function of the $e$-th grey category

The evaluation index $U_{i}$ belongs to the grey evaluation coefficient of the $e$-th grey category

The evaluation index $U_{i}$ belongs to the total grey evaluation coefficient of each grey category

The grey evaluation weight for the $s$-th evaluation equipment of the $e$-th grey category

The grey evaluation weight vector for the $s$-th evaluation equipment of each grey category The grey evaluation weight matrix of the evaluation index $U$ for the $s$-th evaluated equipment

The comprehensive evaluation result of $U$ for the $s$-th evaluated equipment

The comprehensive evaluation value of the $s$ th evaluated equipment

A preventive maintenance replacement cycle of the equipment

The optimal number of PM

The PM cycle

The number of BM in each PM cycle 
The virtual age reduction factor for the $i$-th PM

$\eta$ and $q$ : The cost adjustment factor and the maintenance time adjustment factor $(i+c)^{v}:$ The Stanford-B learning curve: $c$ is the experience parameter and $v$ is an adjustment factor for the maintenance learning effect

$\varphi: \quad$ The experience curve percentage

$z: \quad$ The PM parameter

$\psi_{i}: \quad$ The equipment recovery after the $i$-th PM

$\mu_{i}$ : The incremental failure rate factor of the $i$-th PM

$f_{1}, f_{2}$ and The adjustment factors of the incremental

$g_{1}, g_{2}: \quad$ failure rate

$\beta$ and $\alpha$ : The shape parameter and the scale parameter in the two-parameter Weibull distribution

$T_{w}$ and $T_{d}$ : The normal working time and the downtime of the equipment

A: $\quad$ The equipment availability in a preventive maintenance replacement cycle

$C_{\mathrm{mr}}$ and $t_{\mathrm{mr}}$ : The cost and the average maintenance time for BM

$C_{\mathrm{pm}}$ and $t_{\mathrm{pm}}$ : The cost and the average maintenance time for PM

$C_{r}$ and $t_{r}$ : The cost and the average duration time for RM

$C_{d}$ and $C_{f}$ : The downtime cost and the fixed cost

$C_{p}$ and $\delta: \quad$ The penalty cost and the probability of penalty cost incurred by overtime maintenance

$C_{\mathrm{tm}}, C_{\mathrm{tp}}$, and The total BM, PM, and RM cost

$C_{\text {tr }}$ :

C: $\quad$ The total cost of a preventive maintenance replacement cycle of the equipment

$R_{0}$ : The reliability threshold value.

\section{Data Availability}

The data used to support the findings of this study are included within the article.

\section{Conflicts of Interest}

The authors declare that they have no conflicts of interest.

\section{Acknowledgments}

This research was supported by National Science and Technology Major Project of China (Grant no. 2014ZX04015031), Science and Technology Development Plan Project of Jilin Province (Grant no. 20180520068JH), and Project of Shenzhen Technology University (Grant no. 202018666601007).

\section{References}

[1] H. Su, M. Yang, and Z. Wen, "Multi-layer multi-index comprehensive evaluation for dike safety," Water Resources Management, vol. 29, no. 13, pp. 4683-4699, 2015.
[2] W. Fan, X.-S. Wei, Y.-B. Cao, and B. Zheng, "Landslide susceptibility assessment using the certainty factor and analytic hierarchy process," Journal of Mountain Science, vol. 14, no. 5, pp. 906-925, 2017.

[3] M. N. Cheshmidari, A. H. Hatefi Ardakani, H. Alipor, and S. Shojaei, "Applying Delphi method in prioritizing intensity of flooding in ivar watershed in Iran," Spatial Information Research, vol. 25, no. 2, pp. 173-179, 2017.

[4] B. Liu, F. Zhang, W. Wan, and X. Luo, "Multi-objective decision-making for the ecological operation of built reservoirs based on the improved comprehensive fuzzy evaluation method," Water Resources Management, vol. 33, no. 11, pp. 3949-3964, 2019.

[5] E. Banguero, A. Correcher, Á. Pérez-Navarro, E. García, and A. Aristizabal, "Diagnosis of a battery energy storage system based on principal component analysis," Renewable Energy, vol. 146, pp. 2438-2449, 2020.

[6] E. N. C. Perera, D. T. Jayawardana, P. Jayasinghe, and M. Ranagalage, "Landslide vulnerability assessment based on Entropy method: a case study from kegalle district, Sri Lanka," Modeling Earth Systems and Environment, vol. 5, no. 4, pp. 1635-1649, 2019.

[7] X. Zhu, F. Wang, C. Liang, J. Li, and X. Sun, "Quality credit evaluation based on TOPSIS: evidence from air-conditioning market in China," Procedia Computer Science, vol. 9, pp. 1256-1262, 2012.

[8] X. Wang and Q. Q. Duan, "Improved AHP-TOPSIS model for the ComprehensiveRisk evaluation of oil and gas pipelines," Petroleum Science, vol. 16, no. 1, pp. 1479-1492, 2019.

[9] L. Li, F. Liu, and C. Li, "Customer satisfaction evaluation method for customized product development using Entropy weight and analytic hierarchy process," Computers \& Industrial Engineering, vol. 77, pp. 80-87, 2014.

[10] X. Zhou, L. Xi, and J. Lee, "Reliability-centered predictive maintenance scheduling for A continuously monitored system subject to degradation," Reliability Engineering \& System Safety, vol. 92, no. 4, pp. 530-534, 2007.

[11] S. H. Sheu and C. C. Chang, "An extended periodic imperfect preventive maintenance model with age-dependent failure type," IEEE Transactions on Reliability, vol. 58, no. 2, pp. 397-405, 2011.

[12] Z.-L. Lin, Y.-S. Huang, and C.-C. Fang, "Non-periodic preventive maintenance with reliability thresholds for complex repairable systems," Reliability Engineering \& System Safety, vol. 136, pp. 145-156, 2015.

[13] L. Yang, Z.-S. Ye, C.-G. Lee, S.-F. Yang, and R. Peng, "A twophase preventive maintenance policy considering imperfect repair and postponed replacement," European Journal of Operational Research, vol. 274, no. 3, pp. 966-977, 2019.

[14] T. B. Xia, Y. F. Dong, L. Xiao et al., "Recent advances in prognostics and health management for advanced manufacturing paradigms," Reliability Engineering \& System Safety, vol. 178, pp. 255-268, 2018.

[15] T. B. Xia, X. L. Fang, N. Gebraeel et al., "Online analytics framework of sensor-driven prognosis and opportunistic maintenance for mass customization," Journal of Manufacturing Science and Engineering, vol. 141, no. 5, Article ID 051011, 2019.

[16] T. Xia, B. Sun, Z. Chen, E. Pan, H. Wang, and L. Xi, "Opportunistic maintenance policy integrating leasing profit and capacity balancing for serial-parallel leased systems," Reliability Engineering \& System Safety, vol. 205, Article ID 107233, 2021. 
[17] M. A. K. Malik, "Reliable preventive maintenance scheduling," A I I E Transactions, vol. 11, no. 3, pp. 221-228, 1979.

[18] T. Nakagawa, "Sequential imperfect preventive maintenance policies," IEEE Transactions on Reliability, vol. 37, no. 3, pp. 295-298, 1988.

[19] L. F. Xi, X. J. Zhou, and J. Li, "Research on sequential preventive maintenance policy in finite time horizon," Computer Integrated Manufacturing Systems, vol. 11, no. 10, pp. 14651468, 2005.

[20] Y. C. Yang and P. He, "Flexible cycle preventive maintenance model based on reliability constraint," Journal of Huanggang Normal University, vol. 32, no. 3, pp. 10-14, 2012.

[21] Y. P. Zhang, K. X. Yang, and L. Shi, "Economic optimization model for incomplete preventive maintenance under reliability constraints," Computer Integrated Manufacturing Systems, vol. 24, no. 12, pp. 3019-3026, 2018.

[22] Z. Wang, J. Yang, G. Wang, and G. Zhang, "Sequential imperfect preventive maintenance policy with random maintenance quality under reliability limit," Proceedings of the Institution of Mechanical Engineers, Part C: Journal of Mechanical Engineering Science, vol. 225, no. 8, pp. 1926-1935, 2011.

[23] J.-H. Lim and D. H. Park, "Optimal periodic preventive maintenance schedules with improvement factors depending on number of preventive maintenances," Asia-Pacific Journal of Operational Research, vol. 24, no. 1, pp. 111-124, 2007.

[24] Y. Qu and S. Wu, "Phasic sequential preventive maintenance policy based on imperfect maintenance for deteriorating systems," Journal of Mechanical Engineering, vol. 47, no. 10, pp. 164-170, 2011.

[25] M. L. Zhuo, E. S. Pan, W. Z. Liao et al., "Study on periodic preventive maintenance model based on limitation of reliability," Industrial Engineering and Management, vol. 14, no. 1, pp. 62-65, 2009.

[26] B. Xu, Z. Wang, W. Luo, and T. Ma, "Classification method of production system equipment importance based on grey correlation interval AHP-Entropy method," Journal of Advanced Mechanical Design, Systems, and Manufacturing, vol. 14, no. 6, 2020.

[27] J. Xiao, C. S. Wang, and M. Zhou, "An IAHP-based madm method in urban power system planning," Proceedings of the CSEE, vol. 4, pp. 54-61, 2004.

[28] X. D. Xiao, "Evaluation of digital library collection based on interval Entropy weight TOPSIS method," Theory \& Application, vol. 39, no. 12, pp. 99-102, 2016.

[29] C. R. Zhang, E. Y. Zhu, and J. F. Cheng, "Application of uncertain type of AHP on monorail bridge condition assessment," Journal of Beijing Jiaotong University, vol. 34, no. 6, pp. 16-20, 2010.

[30] X. Liu and B. Chen, "Based on gray comprehensive evaluation method of real estate," Systems Engineering Procedia, vol. 1, pp. 189-194, 2011.

[31] P. Wang, "Evaluation of material management in railway transport enterprise based on grey evaluation method," Railway Transport and Economy, vol. 39, no. 6, pp. 70-75, 2017.

[32] C. Cassady, W. Murdock, E. Pohl et al., "Selective maintenance for support equipment involving multiple maintenance actions," European Journal of Operational Research, vol. 129, no. 2, pp. 252-258, 2001. 